\title{
Management of functional neuroendocrine tumors of the pancreas
}

\author{
Kjell Öberg \\ Department of Medical Sciences, Endocrine Oncology, Uppsala University Hospital, Uppsala, Sweden \\ Correspondence to: Kjell Öberg, MD, PhD. Department of Medical Sciences, Endocrine Oncology, Uppsala University Hospital, Entrance 40:5, SE- \\ 751 85, Uppsala, Sweden. Email: kjell.oberg@medsci.uu.se.
}

\begin{abstract}
Pancreatic neuroendocrine tumors (pNETs) constitute a heterogenous group of malignancies with varying clinical presentation, tumor biology and prognosis. The incidence of pNETs has steadily increased during the last decades with an estimated incidence 2012 of 4.8/100,000. Recent whole genome sequencing of pNETs has demonstrated mutations in the DNA repair genes MUTYH and point mutations and gene fusions in four main pathways from chromatin remodeling, DNA damage repair, activation of mechanistic target of rapamycin (mTOR) signaling and the telomere maintenance. This new information will be the foundation for new therapies in the near future for malignant pNETs. The functioning pNETs constitute about $30-40 \%$ of all pNETs displaying nine different clinical syndromes: insulinoma, ZollingerEllison, Verner-Morrison, glucagonoma, somatostatinomas, ectopic adrenocorticotropic hormone (ACTH) and parathyroid hormone related peptide (PTH-rP) syndromes. Single patients might also present carcinoid syndrome. The diagnostic work-up include histopathology with the new WHO 2017 Classification, biomarkers (CgA, NSE), radiology and molecular imaging including CT-scan, magnetic resonance imaging (MRI), ultrasound and PET-scan. A cornerstone in the treatment of pNETs is surgery which is rarely curative but can reduce the clinical symptoms by debulking which also include radiofrequency ablation, embolization of liver metastases. Medical treatment includes chemotherapy and the targeted agents such as everolimus, sunitinib and peptide receptor radiotherapy (PRRT). Somatostatin analogs has for the last decades been the main stay for management for clinical symptoms related to functioning pNETs and is often combined with new targeted agents as well as chemotherapy. Long-term management of functioning pNETs need a combination of different procedures, surgery, local ablation, targeted agents and somatostatin analogs. Future therapies might be based on the recent advances in molecular genetics and tumor biology.
\end{abstract}

Keywords: Functioning pancreatic neuroendocrine tumors (FpNETs); positron emission tomography scan (PET scan); chemotherapy; targeted agents; everolimus; sunitinib; peptide receptor radiotherapy (PRRT); surgery

Submitted Oct 05, 2017. Accepted for publication Oct 26, 2017.

doi: 10.21037 /gs.2017.10.08

View this article at: http://dx.doi.org/10.21037/gs.2017.10.08

\section{Introduction}

Neuroendocrine tumors of the pancreas are a heterogenous, malignant disease with varying tumor biology and clinical presentation. The annual incidence of all pancreatic neuroendocrine tumors (pNETs) is $0.8 / 100,000$, that includes both functioning and non-functioning pNETs (1). Non-function tumors contribute $60 \%$ of all pNETs. The median overall survival of localized disease is 250 months, for regional disease about 9 years and for distant metastases 25 months (1). The genetic background has been unsolved for many years but recently a paper presented a whole genome sequencing of 102 sporadic pancreatic NETs, which showed some new and interesting findings. Clinically, sporadic pNETs contain a larger than expected proportion of germ line mutations, including previously unreported mutations in the DNA repair genes (MUTYH), CHEK2 and BRCA2. Together with mutations in the MEN1 and VHL, these occur in $17 \%$ of the patients. Somatic mutations including point mutations and gene fusions were commonly found in genes involved in four main pathways; 
chromatin remodeling, DNA damage repair, activation of mechanistic target of rapamycin (mTOR) signaling and telomere maintenance. In addition, a subgroup that was identified and related with hypoxia, and HIF signaling (2). This genetic information will of course give important data for future therapies. About 30-40\% of patients with pNETs present, a clinical syndrome related to a specific hormone excess state (3).

The diagnosis is based on histopathology, biomarkers and radiology. The pathology report should contain information about specific staining for chromogranin A, synaptophysin and the proliferation marker Ki-67 (4,5). Based on proliferation (Ki-67) the tumors are divided into NET G1 (Ki-67 <3\%), NET G2 (Ki-67, 3-20\%), NET G3 (Ki-67 >20\%) and neuroendocrine carcinoma (NEC) G3 (Ki-67 $>20 \%$, poorly differentiated). mixed adenoneuroendocrine carcinoma (MiNEN) (WHO 2017) $(6,7)$.

Circulating biomarkers include chromogranin A, neuron specific enolase (NSE) as general markers and specific markers are plasma gastrin, insulin, pro-insulin, glucagon, vasoactive intestinal peptide (VIP), adrenocorticotropic hormone (ACTH), parathyroid hormone related peptide (PTH-rP) usually taken in relation to clinical symptoms (see later) $(8,9)$. Radiology include standard CT-scans, ultrasound and magnetic resonance imaging (MRI) but nowadays positron emission tomography (PET) combined with CT-scan or MRI. Ga68DOTATATE/TOC/CT is today the most sensitive diagnostic and staging procedure. Other isotopes are C11-5HTP or F18-DOPA. Somatostatin receptor scintigraphy is replaced in most countries by Ga68-DOTATATE PET/CT-scans (10). Endoscopic ultrasonography is another sensitive method to localize the primary tumor in the pancreas (11).

The treatment of functioning pancreatic neuroendocrine tumors (FpNETs) has at least two goals; one to control the clinical symptoms and the hormone excess but also the tumor growths, which can sometimes be rather aggressive and rapid. There are ten well-established pNETs syndromes, which nine are associated with functional syndromes. The last one is non-functioning tumors which in a strict sense are not non-functional due to secretion of a number of different products; chromogranin A, NSE, pancreatic polypeptide, ghrelin, neurotonin and so forth $(3,12)$. These agents are not resulting in any specific clinical syndrome. The nine functional pNETs include; gastrinomas releasing gastrin with the clinical syndrome, called Zollinger-Ellison syndrome, insulinomas, Verner Morrison syndrome (VIPomas), secreting vasoactive intestinal peptide, glucagonomas, somatostatinomas, GRFomas, secreting growths hormone releasing factor ACTHomas and pNET secreting serotonin causing carcinoid syndrome and pNETs causing hypercalcemia by secreting PTHrP $(3,12)$. In addition to these well-established pNET syndromes, there are pNET secreting luteinizing hormone, renin, GLP1, IGF1, erythropoietin, enteroglucagon and cholecystokinin (12-14). These syndromes are not discussed in this review since they are very rare with just single patients in each group. pNET syndromes caused entirely by NETs in the pancreas are insulinoma and glucagonoma. Other syndromes that classifies as pNET syndromes can occur in NETs at extra pancreatic sites. For example, duodenal gastrinoma causing Zollinger Ellison syndrome, duodenal somatostatinomas associated with somatostatinoma syndrome. VIPomas occurring in neural tissue (ganglioneuroma) and GRFomas occurring in the small intestine $(12,15)$. This article will focus on recent advances as well as controversies in the management of functional pNETs. Most of the reports on an efficacy of the advances in control of hormone excess stage are reported in case series, case reports or retrospective studies. In addition, symptom control is frequently not the primary endpoint and the studies are usually single center studies. For each of the FpNETs, the hormone excess state needs to be controlled as quickly as possible sometimes before the diagnosis and staging of the disease is established. Control of hormone excess is necessary during tumor localization and this procedure can be time consuming and be difficult in some of the FpNETs (16). The primary tumor can be small and therefore not localized by conventional cross-sectional imaging (CT, ultrasound, MRI). Recently, positron emission tomography with Gallium68-labled somatostatin analogs as well as C11 labeled 5-hydroxytryptophan (5-HTP) are very sensitive and help in localization of both primary tumor and in the staging of the disease. Endoscopic ultrasound is another sensitive method to localize the primary tumor in the pancreas $(10,11)$.

\section{Treatment of functioning pancreatic neuroendocrine tumors (FpNETs) in general}

Numerous studies have demonstrated that cytoreductive surgery can facilitate the management of hormone excess state and should therefore be considered in any patients with increasing refractory symptoms. The general opinion is that cytoreductive surgery or debulking can be considered in patients with $70-90 \%$ of the visible disease is resectable. Despite lack of control studies, the ENETS 
Guidelines recommend surgery for curative intent whenever possible (16). Unfortunately, only $5-15 \%$ of the patients with FpNETs that have metastatic disease to the liver, have potentially completely resectable disease with a possibility of surgical cure (16). Liver transplantation is the ultimate hepatic debulking procedure and occasionally used in patients with refractory hormone excess stage with the tumor confined to the liver (16). Liver directed therapies include trans arterial embolization (TAE), trans arterial chemoembolization (TACE), radioembolization or selective internal radiotherapy (SIRT) as well as radiofrequency ablation (RFA) (17). TAE and TACE can be safely performed since NET metastases in the liver are highly vascular and derive their blood supply primary from the hepatic artery, whereas the normal liver parenchyma is supplied primarily by the portal vein. The procedure is performed percutaneously in most cases and only occlusion (TAE) or with co-administration of chemotherapeutic agents (TACE, usually doxorubicin, streptozotocin, fluorouracil or cisplatinum). The procedures can reduce the hepatic tumor mass to $80 \%$ of the patients with the duration of up to 45 months (18). Radiofrequency ablation (SIRT) involves use of Yttrium-90 (Y-90) microspheres with either resin microspheres (SIR-Spheres) or Y-90 glass microspheres (Thera-Spheres). The procedure can be repeated taken one lobe at the time. The response rates have been up to $75-80 \%$ and lasting for 20-40 months (17). Radiofrequency ablation (RFA) mostly performed in combination with surgery, especially to remove isolated metastases. Besides the use of RFA as an anti-tumor treatment, a number of studies have reported enhanced symptomatic control of FpNETs $(19,20)$.

\section{Medical treatment of pNETs}

Streptozotocin, an alkylating agent that has been used for more than 50 years in the treatment of functioning pNETs. The first case being an insulin producing tumor thereafter, multiple studies of streptozotocin alone or in combination with 5-FU or doxorubicin has demonstrated effective response rates in up to $40 \%$ of the patients and a duration of $>2$ years (21-24). In recent years, treatment with temozolomide plus capecitabine has been applied with response rates up to $70 \%$ in non-randomized trials (25-27). Both cytotoxic treatments are still considered first line treatment by international guidelines (ENETS, NANETS) $(21,28)$. In NEC G3, the recommended treatment is carboplatin/cisplatin plus etoposide (29). Cytotoxic treatment can definitely control clinical symptoms related to hormone production by the pNETs, usually it takes some time to receive significant decrease in circulating hormone levels. Therefore, this kind of treatments are usually combined with somatostatin analogs for more rapid control of clinical symptoms. Biotherapy for NETs emerged in the 1980s when somatostatin analogs and interferons were investigated in small series of patients with malignant carcinoid syndrome. During the past decades, a long-acting, repeatedly depot formulation of octreotide, Sandostatin LAR ${ }^{\circledR}$ have been available as well as a second somatostatin analog, lanreotide with either depot formulation (Somatuline Autogel ${ }^{\circledR}$ ) has been developed for treatment of hormone related symptoms, both GI and pancreatic NETs (30-33). In the recent years, strong evidence has emerged that somatostatin analogs can inhibit the growths of NETs (34-36). Indirect anti proliferative mechanisms include inhibition of circulating growth factors such as, vascular endothelial growth factor as well as inhibition of tumor angiogenesis. Direct mechanism involve of somatostatin receptors binding which inhibit cellular proliferation and mediate apoptosis (37). Definite evidence of growth inhibition of somatostatin analogs at doses of $20-30 \mathrm{mg}$ for octreotide LAR and 90-120 $\mathrm{mg}$ for Somatuline Autogel, reported in two randomized phase III trials $(34,35)$. Somatostatin analogs can control clinical symptoms in up to $60 \%$ of patients with functioning tumors (37). In patients refractory to standard doses of somatostatin analog, high-dose treatment has been attempted by either shortened the interval between the injections every 2-4 weeks and increasing the dose of octreotide up to $60 \mathrm{mg}$, lanreotide up to $180 \mathrm{mg}$ can completely or partially control the clinical symptoms in about $40-60 \%$ of the patients $(38,39)$. Pasireotide is a novel somatostatin analog binding to $4 / 5$ somatostatin receptors. The analog has particularly been applied in patients with the carcinoid syndrome, with a significant reduction of diarrhea and flushing in about $30 \%$ of the patients, as well as in patients with functioning pNETs and the Cushing's syndrome due to ACTH-production $(38,40)$. Interferons $(\mathrm{IFN}-\alpha)$ might exert a direct anti proliferative effect on tumor growths besides reducing circulating hormone levels. Interferon has been used in G1 NETs of the small intestinal tract with biochemical responses in up to $60 \%$, tumor response in $9-10 \%$ $(41,42)$. Interferon has been applied in patients with FpNETs, in particular VIP-producing tumors refractory to somatostatin analogs showing control of symptoms 
and hormone levels for extended periods of time (41). Several studies have evaluated the combination of somatostatin analogs and interferon, indicating some additional benefit by combining the both (41).

\section{Targeted agents}

The mTOR-inhibitor everolimus is registered worldwide for treatment of pNETs as well as GI and lung-NETs in patients with advanced progressive disease $(43,44)$. Two placebo controlled prospective randomized phase III studies have supported the results for everolimus significantly prolonged the progression free survival in each of these groups of patients $(43,44)$. Everolimus is also effective in controlling the hormone excess state in patients with refractory, functioning NET syndromes. It has been particularly useful for treatment of refractory malignant insulin producing tumors to control the hypoglycemia and in VIP-producing pNETs $(45,46)$. The tyrosine kinase inhibitor, sunitinib that inhibits tyrosine kinase activity of PDGFRs, VGFR1, VGFR2, cKIT and FRT3 is approved worldwide for the treatment of pNETs (47). The approval was based on the results of a double blind, prospective placebo controlled, phase III study, which demonstrated that sunitinib extended the progression free survival in these patients, as well as increased over all survival. Sunitinib is in principal used for antiproliferation in patients with pNETs and it also reported to effectively control hormone excess in patients with refractory symptoms and pNET syndromes $(48,49)$. Another tyrosine kinase inhibitor, pazopanib as well as cabozantinib has demonstrated some activity in pNETs, both for antiproliferation and hormone control.

\section{Peptide receptor radiotherapy (PRRT) with 177-Lutetium labelled somatostatin analogs.}

Studies of PRRT using radio labelled somatostatin analogs have demonstrated a clear anti-tumor efficacy in patients with both GI and pNETs. PRRT was recently approved by EMA for treatment of all types of NETs based on the NETTER-1 trial and data from several phase II trials $(50,51)$. A recent study in small intestinal NETs indicated a significant anti-tumor response would certainly result in an approval in the US quite soon. PRRT has been considerably effective in controlling hormone excess state in patients with refractory FpNET syndromes (52-54).

\section{Clinical syndromes}

\section{Insulinoma}

In more than $90 \%$ of patients with insulinoma, the treatment is curative by surgical resection. The tumors are usually solitary and benign $(15,16)$. In about $3 \%$ of the patients, insulinomas occur in patients with multiple endocrine neoplasia type 1 (MEN1) in which they can be recurrent and multiple (55). The control of the hormone excess in patients with insulinomas is usually only needed during the preoperative period as well as in the group of patients with malignant insulinomas. The primary treatment has been glucose infusions with frequent small feedings and the use of diazoxide, which can control symptoms in $50-60 \%$ of the patients (56). Somatostatin analogs are also used and are effective in about $50 \%$ of the patients (57). However, in some patients they can worsen the symptoms by inhibiting counter regulatory mechanisms such as glucagon/growth hormone release (58). In patients with malignant insulinomas $(<10 \%$ of all cases), the hormone excess can be difficult to control. Treatment with everolimus has demonstrated significant efficacy, other treatment options has been with the tyrosine kinase inhibitor, sunitinib as well as peptide receptor radiotherapy (PRRT) $(45,46,48,49,51)$. Liver directed therapy including TACE/TAE, radioembolization, radiofrequency ablation (RFA), ablated treatment such as ethanol or microwave of metastases has been effective in many instances. Increasing doses of octreotide/lanreotide in refractory cases can be of value $(17,18,38,39)$.

\section{Zollinger-Ellison syndrome}

The majority of patients with Zollinger-Ellison syndrome require life-long treatment of the gastric acid hypersecretion. Only $25-35 \%$ of the patients with Zollinger-Ellison are cured long-term by surgery. About $25 \%$ of the patients have MEN1 with multiple small gastrinomas frequently with lymph node metastases (59-61). The symptomatic control of gastric acid hypersecretion is highly effective with proton pumping inhibitors (PPIs) or histamine $\mathrm{H} 2$ receptor antagonists $(60,62)$. Somatostatin analogs are also effective but rarely used because they require parental administration compare to the orally active PPIs and histamine $\mathrm{H} 2$ receptor antagonist (62). Antitumor treatment includes everolimus, sunitinib, PRRT, liver directed therapies including TACE/TAE and radioembolization. 
Verner-Morrison syndrome (VIPoma syndrome)

Patients with Verner-Morrison syndrome are rarely cured by surgery due to presentation of metastatic disease at diagnosis. Therefore, life-long treatment of hormone excess is usually required $(3,59)$. The main stay of treatment is the use of somatostatin analogs octreotide/lanreotide which controls the large volume diarrhea in $70-90 \%$ of the patients, although, some patients become refractory $(15,16)$. Alpha interferon has also demonstrated a significant anti-secretory effect in patients with Verner-Morrison syndrome refractory to somatostatin analog (41). Antitumoral therapy such as everolimus and sunitinib can also control the secretory state as well as PRRT. Liver directed therapies including TACE/TEA, radioembolization, radiofrequency ablation can control the disease for various periods of time. By increasing the dose of octreotide/lanreotide in refractory cases, control of the disease can be obtained temporarily.

\section{Glucagonomas}

The majority of patients with glucagon producing tumors (glucagonomas) present metastatic disease. Somatostatin analog can control many of the symptoms related to the syndrome, such as the skin rash, diabetes and thromboembolism (37). Newer therapies such as everolimus and sunitinib is of course of value together with PRRT and liver targeted treatments. PRRT is also very effective in controlling the clinical symptoms as well as control of the tumor growth.

\section{ACTH-producing pNETs}

Ectopic Cushing's syndrome is due to pancreatic ACTHproducing tumors in up to $16 \%$ of the cases. Cushing's syndrome can be the only sign of a functional pNET and occurs in $3-4 \%$ of patients with Zollinger-Ellison syndrome (63). It usually is a development associated with a poor prognosis since these tumors mostly are aggressive. In a large number of cases, bilateral adrenalectomy is the only way to control the clinical symptoms. The hypercortisolemia can be controlled by metyrapone and ketoconazole. Recently, other classes of drugs have been used and been reported to be effective including gluco cortical receptor antagonist (mifepristone). Adrenal specific therapy; etomidate, osilodrostat and fluconazole $(63,64)$. Other agents are somatostatin analogs including pasireotide (38), dopamine receptor agonist cabergoline. In addition, PRRT has been reported to be of help in controlling the hypercortisolemia.

\section{General comments}

Somatostatin analogs as well as gastric acid anti-secretory agents, PPIs and $\mathrm{H} 2$ receptor antagonist have made it possible to control hormone excess states in many subtypes of FpNETs. However, the control of hormone excess stage can be particularly difficult in patients with insulin and VIP as well as ACTH-producing pNETs. Therefore, additional treatment is necessary with targeted agents like everolimus and sunitinib as well as PRRT. Patients with FpNETs have two problems regarding management, antitumor treatment and treatment of the hormone excess state. The antitumor treatments, for example chemotherapy and targeted agents, while effective against tumor growth, they may not control the hormone excess state. Therefore, combined therapies might be necessary to be able to manage these difficulties. The long-term treatment of hormone excess state remains an obstacle in many patients due to drug resistance developing with time, particularly in patients with malignant insulinomas, VIPomas and pancreatic ACTHomas. A combination of cytotoxic therapy such as streptozotocin plus 5-FU, temozolomide plus capecitabine together with somatostatin analogs, are able to control FpNETs for very long periods. PRRT is another option and can be combined with somatostatin analogs in controlling clinical symptoms long-term. The targeted agents like everolimus, sunitinib as well as PRRT were originally developed for control of tumor growth. As an additional effect, it was also noticed a clear reduction in secreted hormones and thereby improvement of clinical symptoms. Telotristat ethyl was recently registered for treatment of patients with the carcinoid syndrome depending on excess of serotonin secretion from the tumor (65). There are a small number of pNETs with serotonin production and signs of carcinoid syndrome which might be treated with this new compound (66). High dose treatment with somatostatin analogs is now in different clinical trials, mostly with octreotide (40-60 mg/4 w) and lanreotide (180 mg/4 w) which might give a clinical benefit to patients with refractory symptoms to standard doses of somatostatin analogs. For the moment, most attention is focusing on preventing tumor growth. Therefore, no particular new agents only controlling 
hormone secretion and clinical symptoms are viewable in the developing pipelines at various pharmaceutical companies.

\section{Acknowledgements}

None.

\section{Footnote}

Conflicts of Interest: The author has no conflicts of interest to declare.

\section{References}

1. Dasari A, Shen C, Halperin D, et al. Trends in the incidence, prevalence, and survival outcomes in patients with neuroendocrine tumors in the United States. JAMA Oncol 2017;3:1335-42.

2. Scarpa A, Chang DK, Nones K, et al. Whole-genome landscape of pancreatic neuroendocrine tumours. Nature 2017;543:65-71.

3. Jensen R, Norton JA, Oberg K. Neuroendocrine Tumors. In: Feldman M, Friedman LS, Brandt LJ. editors. Sleisenger and Fordtran's Gastrointestinal and Liver Disease. Philadelphia: Elsevier Health Sciences; 16 A.D., 2015:501-41.

4. Klimstra DS, Modlin IR, Adsay NV, et al. Pathology reporting of neuroendocrine tumors: application of the Delphic consensus process to the development of a minimum pathology data set. Am J Surg Pathol 2010;34:300-13.

5. Perren A, Couvelard A, Scoazec JY, et al. ENETS Consensus Guidelines for the Standards of Care in Neuroendocrine Tumors: Pathology: Diagnosis and Prognostic Stratification. Neuroendocrinology 2017;105:196-200.

6. Lloyd RV, Osamura RY, Klöppel G. editors. WHO Classification of Tumours of Endocrine Organs, Chapter 6, (210-239) 4th ed. Lyon: International Agency for Research on Cancer, 2017.

7. Kloppel G, Rindi G, Perren A, et al. The ENETS and AJCC/UICC TNM classifications of the neuroendocrine tumors of the gastrointestinal tract and the pancreas: a statement. Virchows Arch 2010;456:595-7.

8. Oberg K. Circulating biomarkers in gastroenteropancreatic neuroendocrine tumours. Endocr Relat Cancer 2011;18 Suppl 1:S17-25.

9. Oberg K, Couvelard A, Delle Fave G, et al.
ENETS Consensus guidelines for standard of care in neuroendocrine tumours: biochemical markers. Neuroendocrinology 2017;105:201-11.

10. Sundin A, Arnold R, Baudin E, et al. ENETS consensus guidelines for the standards of care in neuroendocrine tumors: radiological, nuclear medicine and hybrid imaging. Neuroendocrinology 2017;105:212-44.

11. Anderson MA, Carpenter S, Thompson NW, et al. Endoscopic ultrasound is highly accurate and directs management in patients with neuroendocrine tumors of the pancreas. Am J Gastroenterol 2000;95:2271-7.

12. Ito T, Igarashi H, Jensen RT. Pancreatic neuroendocrine tumors: clinical features, diagnosis and medical treatment: advances. Best Pract Res Clin Gastroenterol 2012;26:737-53.

13. Maton PN, Gardner JD, Jensen RT. Cushing's syndrome in patients with the Zollinger-Ellison syndrome. $\mathrm{N} \mathrm{Engl} \mathrm{J}$ Med 1986;315:1-5.

14. Rehfeld JF, Federspiel B, Bardram L. A neuroendocrine tumor syndrome from cholecystokinin secretion. $\mathrm{N}$ Engl J Med 2013;368:1165-6.

15. Jensen RT, Cadiot G, Brandi ML, et al. ENETS Consensus Guidelines for the management of patients with digestive neuroendocrine neoplasms: functional pancreatic endocrine tumor syndromes. Neuroendocrinology 2012;95:98-119.

16. Partelli S, Bartsch DK, Capdevila J, et al. ENETS consensus guidelines for standard of care in neuroendocrine tumours: surgery for small intestinal and pancreatic neuroendocrine tumours. Neuroendocrinology 2017;105:255-65.

17. Kennedy AS. Hepatic-directed therapies in patients with neuroendocrine tumors. Hematol Oncol Clin North Am 2016;30:193-207.

18. de Baere T, Deschamps F, Tselikas L, et al. GEP-NETS update: Interventional radiology: role in the treatment of liver metastases from GEP-NETs. Eur J Endocrinol 2015;172:R151-66.

19. Eriksson J, Stalberg P, Nilsson A, et al. Surgery and radiofrequency ablation for treatment of liver metastases from midgut and foregut carcinoids and endocrine pancreatic tumors. World J Surg 2008;32:930-8.

20. Mazzaglia PJ, Berber E, Milas M, et al. Laparoscopic radiofrequency ablation of neuroendocrine liver metastases: a 10-year experience evaluating predictors of survival. Surgery 2007;142:10-9.

21. Pavel M, Valle JW, Eriksson B, et al. ENETS consensus guidelines for the standards of care in neuroendocrine 
neoplasms: systemic therapy - biotherapy and novel targeted agents. Neuroendocrinology 2017;105:266-80.

22. Murray-Lyon IM, Eddleston AL, Williams R, et al. Treatment of multiple-hormone-producing malignant isletcell tumour with streptozotocin. Lancet 1968;2:895-8.

23. Moertel CG, Lefkopoulo M, Lipsitz S, et al. Streptozocindoxorubicin, streptozocin-fluorouracil or chlorozotocin in the treatment of advanced islet-cell carcinoma. N Engl J Med 1992;326:519-23.

24. Kouvaraki MA, Ajani JA, Hoff P, et al. Fluorouracil, doxorubicin, and streptozocin in the treatment of patients with locally advanced and metastatic pancreatic endocrine carcinomas. J Clin Oncol 2004;22:4762-71.

25. Ekeblad S, Sundin A, Janson ET, et al. Temozolomide as monotherapy is effective in treatment of advanced malignant neuroendocrine tumors. Clin Cancer Res 2007;13:2986-91.

26. Strosberg JR, Fine RL, Choi J, et al. First-line chemotherapy with capecitabine and temozolomide in patients with metastatic pancreatic endocrine carcinomas. Cancer 2011;117:268-75.

27. Walter T, van Brakel B, Vercherat C, et al. O6Methylguanine-DNA methyltransferase status in neuroendocrine tumours: prognostic relevance and association with response to alkylating agents. Br J Cancer 2015;112:523-31.

28. Garcia-Carbonero R, Rinke A, Valle JW, et al. ENETS consensus guidelines for the standards of care in neuroendocrine neoplasms. systemic therapy 2: chemotherapy. Neuroendocrinology 2017;105:281-94.

29. Garcia-Carbonero R, Sorbye H, Baudin E, et al. ENETS consensus guidelines for high-grade gastroenteropancreatic neuroendocrine tumors and neuroendocrine carcinomas. Neuroendocrinology 2016;103:186-94.

30. Pavel M, O'Toole D, Costa F, et al. ENETS consensus guidelines update for the management of distant metastatic disease of intestinal, pancreatic, bronchial neuroendocrine neoplasms (NEN) and NEN of Unknown Primary Site. Neuroendocrinology 2016;103:172-85.

31. Oberg K. Interferon-alpha versus somatostatin or the combination of both in gastro-enteropancreatic tumours. Digestion 1996;57 Suppl 1:81-3.

32. Oberg K, Kvols L, Caplin M, et al. Consensus report on the use of somatostatin analogs for the management of neuroendocrine tumors of the gastroenteropancreatic system. Ann Oncol 2004;15:966-73.

33. Wymenga AN, Eriksson B, Salmela PI, et al. Efficacy and safety of prolonged-release lanreotide in patients with gastrointestinal neuroendocrine tumors and hormonerelated symptoms. J Clin Oncol 1999;17:1111.

34. Rinke A, Muller HH, Schade-Brittinger C, et al. Placebocontrolled, double-blind, prospective, randomized study on the effect of octreotide LAR in the control of tumor growth in patients with metastatic neuroendocrine midgut tumors: a report from the PROMID Study Group. J Clin Oncol 2009;27:4656-63.

35. Caplin ME, Pavel M, Cwikla JB, et al. Lanreotide in metastatic enteropancreatic neuroendocrine tumors. N Engl J Med 2014;371:224-33.

36. Jann H, Denecke T, Koch M, et al. Impact of octreotide long-acting release on tumour growth control as a firstline treatment in neuroendocrine tumours of pancreatic origin. Neuroendocrinology 2013;98:137-43.

37. Grozinsky-Glasberg S, Shimon I, Korbonits M, et al. Somatostatin analogues in the control of neuroendocrine tumours: efficacy and mechanisms. Endocr Relat Cancer 2008;15:701-20.

38. Strosberg JR, Benson AB, Huynh L, et al. Clinical benefits of above-standard dose of octreotide LAR in patients with neuroendocrine tumors for control of carcinoid syndrome symptoms: a multicenter retrospective chart review study. Oncologist 2014;19:930-6.

39. Al-Efraij K, Aljama MA, Kennecke HF. Association of dose escalation of octreotide long-acting release on clinical symptoms and tumor markers and response among patients with neuroendocrine tumors. Cancer Med 2015;4:864-70.

40. Schmid HA. Pasireotide (SOM230): development, mechanism of action and potential applications. Mol Cell Endocrinol 2008;286:69-74.

41. Oberg K. Interferon in the management of neuroendocrine GEP-tumors: a review. Digestion 2000;62 Suppl 1:92-7.

42. Ferrantini M, Capone I, Belardelli F. Interferon-alpha and cancer: mechanisms of action and new perspectives of clinical use. Biochimie 2007;89:884-93.

43. Yao JC, Shah MH, Ito T, et al. Everolimus for advanced pancreatic neuroendocrine tumors. N Engl J Med 2011;364:514-23.

44. Yao JC, Fazio N, Singh S, et al. Everolimus for the treatment of advanced, non-functional neuroendocrine tumours of the lung or gastrointestinal tract (RADIANT-4): a randomised, placebo-controlled, phase 3 study. Lancet 2016;387:968-77.

45. Fiebrich HB, Siemerink EJ, Brouwers AH, et al. Everolimus induces rapid plasma glucose normalization in insulinoma patients by effects on tumor as well as normal tissues. Oncologist 2011;16:783-7. 
46. Thomas NJ, Brooke AM, Besser GM. Long-term maintenance of normoglycaemia using everolimus in a patient with disseminated insulinoma and severe hypoglycaemia. Clin Endocrinol (Oxf) 2013;78:799-800.

47. Raymond E, Dahan L, Raoul JL, et al. Sunitinib malate for the treatment of pancreatic neuroendocrine tumors. $\mathrm{N}$ Engl J Med 2011;364:501-13.

48. de Mestier L, Walter T, Brixi H, et al. Sunitinib achieved fast and sustained control of VIPoma symptoms. Eur J Endocrinol 2015;172:K1-3.

49. Chen J, Wang C, Han J, et al. Therapeutic effect of sunitinib malate and its influence on blood glucose concentrations in a patient with metastatic insulinoma. Expert Rev Anticancer Ther 2013;13:737-43.

50. Hicks RJ, Kwekkeboom DJ, Krenning E, et al. ENETS consensus guidelines for the standards of care in neuroendocrine neoplasia: peptide receptor radionuclide therapy with radiolabeled somatostatin analogues. Neuroendocrinology 2017;105:295-309.

51. Strosberg J, El-Haddad G, Wolin E, et al. Phase 3 Trial of 177Lu-Dotatate for midgut neuroendocrine tumors. N Engl J Med 2017;376:125-35.

52. Kwekkeboom DJ, Kam BL, van Essen M, et al. Somatostatin-receptor-based imaging and therapy of gastroenteropancreatic neuroendocrine tumors. Endocr Relat Cancer 2010;17:R53-73.

53. van Schaik E, van Vliet EI, Feelders RA, et al. Improved control of severe hypoglycemia in patients with malignant insulinomas by peptide receptor radionuclide therapy. $\mathrm{J}$ Clin Endocrinol Metab 2011;96:3381-9.

54. Makis W, McCann K, Riauka TA, et al. Ectopic Corticotropin-producing neuroendocrine tumor of the pancreas treated with 177Lu DOTATATE Induction and Maintenance Peptide Receptor Radionuclide Therapy. Clin Nucl Med 2016;41:50-2.

55. Makis W, McCann K, Riauka TA, et al. Glucagonoma Pancreatic Neuroendocrine Tumor Treated With 177Lu DOTATATE Induction and Maintenance Peptide Receptor Radionuclide Therapy. Clin Nucl Med 2015;40:877-9.

56. Jensen RT, Berna MJ, Bingham DB, et al. Inherited pancreatic endocrine tumor syndromes: advances in

Cite this article as: Öberg K. Management of functional neuroendocrine tumors of the pancreas. Gland Surg 2018;7(1):20-27. doi: $10.21037 / g s .2017 .10 .08$ molecular pathogenesis, diagnosis, management, and controversies. Cancer 2008;113:1807-43.

57. Baudin E, Caron P, Lombard-Bohas C, et al. Malignant insulinoma: recommendations for characterisation and treatment. Ann Endocrinol (Paris) 2013;74:523-33.

58. Vezzosi D, Bennet A, Rochaix P, et al. Octreotide in insulinoma patients: efficacy on hypoglycemia, relationships with Octreoscan scintigraphy and immunostaining with anti-sst $2 \mathrm{~A}$ and anti-sst 5 antibodies. Eur J Endocrinol 2005;152:757-67.

59. Healy ML, Dawson SJ, Murray RM, et al. Severe hypoglycaemia after long-acting octreotide in a patient with an unrecognized malignant insulinoma. Intern Med J 2007;37:406-9.

60. Falconi M, Eriksson B, Kaltsas G, et al. ENETS Consensus guidelines update for the management of patients with functional pancreatic neuroendocrine tumors and non-functional pancreatic neuroendocrine tumors. Neuroendocrinology 2016;103:153-71.

61. Jensen RT, Niederle B, Mitry E, et al. Gastrinoma (duodenal and pancreatic). Neuroendocrinology 2006;84:173-82.

62. Norton JA, Alexander HR, Fraker DL, et al. Does the use of routine duodenotomy (DUODX) affect rate of cure, development of liver metastases, or survival in patients with Zollinger-Ellison syndrome? Ann Surg 2004;239:61725; discussion 26.

63. Ito T, Igarashi H, Uehara H, et al. Pharmacotherapy of Zollinger-Ellison syndrome. Expert Opin Pharmacother 2013;14:307-21.

64. Nieman LK, Biller BM, Findling JW, et al. Treatment of Cushing's Syndrome: An Endocrine Society Clinical Practice Guideline. J Clin Endocrinol Metab 2015;100:2807-31.

65. Moraitis AG, Auchus RJ. Mifepristone Improves Octreotide Efficacy in Resistant Ectopic Cushing's Syndrome. Case Rep Endocrinol 2016;2016:8453801.

66. Hörsch D, Kulke M, Caplin M, et al. Efficacy and safety of telotristat etiprate in patients with carcinoid syndrome not adequately controlled by somatostatin analog therapy: Analysis of the ongoing TELESTAR extension period. Neuroendocrinology 2016;103:88-88s. 